\title{
On the Classical Model for Microwave Induced Escape from a Josephson Washboard Potential
}

\author{
James A. Blackburn ${ }^{1}$, Matteo Cirillo ${ }^{2}$, and Niels Grønbech-Jensen ${ }^{3}$ \\ 1) Department of Physics \&5 Computer Science, Wilfrid Laurier University, Waterloo, Ontario N2L 3C5, Canada \\ ${ }^{2)}$ Dipartimento di Fisica and MINAS-Lab, Università di Roma "Tor Vergata", I-00173 Roma, Italy and \\ ${ }^{3)}$ Department of Applied Science, University of California, Davis, California 95616
}

\begin{abstract}
We revisit the interpretation of earlier low temperature experiments on Josephson junctions under the influence of applied microwaves. It was claimed that these experiments unambiguously established a quantum phenomenology with discrete levels in shallow wells of the washboard potential, and macroscopic quantum tunneling. We here apply the previously developed classical theory to a direct comparison with the original experimental observations, and we show that the experimental data can be accurately represented classically. Thus, our analysis questions the necessity of the earlier quantum mechanical interpretation.
\end{abstract}

Since early days, Josephson junctions in superconducting circuits have been modeled very successfully with the so-called Resistively and Capacitively Shunted Junction (RCSJ) equivalent circuit [1]. In the situation where a junction is biased with a fixed current, that equivalent circuit yields an equation of motion for the junction phase that is analogous to a fictitious particle confined to a "washboard" potential consisting of repeated wells. In the presence of thermal noise, a Josephson junction biased just below its critical current may be excited out of its position at the bottom of a well and into a running state with an accompanying voltage. In 1981, a paper appeared 2] which reported experiments carried out on such a current biased Josephson junction, but at much lower temperatures (down to $3 m K$ ) than previously. It was found that at high temperatures the transition rate out of the metastable state was well described by thermal activation over the barrier formed by the rim of the well, but that as $T \rightarrow 0$, the transition rate was dominated by quantum mechanical tunneling of the macroscopic junction phase (MQT). The transition from classical to quantum behavior was understood to occur in the neighborhood of a crossover temperature [3]. A few years later [4], Devoret et al. carried out another such experiment and again saw this evidence for MQT at the lowest temperatures attained.

Beginning in 1985, Martinis, Devoret and Clarke [5, 6] applied these ideas to the interpretation of experiments which added microwaves to the fixed current bias of an isolated Josephson junction. The thinking was that because these experiments were carried out at low temperatures $(18 m K$ and $28 m K)$, only a quantum model need be considered. Biasing the junction very close to its critical current makes the well very shallow. Within the conceptual framework of quantum phenomena, a well must contain discrete levels, and a shallow well will contain only a few of them. The action of properly tuned microwave radiation was then understood to populate the upper level from which escape would occur via macroscopic quantum tunneling of the virtual particle (i.e., the system would switch to the running state). The preferred frequencies for enhanced escape observed in the experiment were thus taken as signatures of the spacing of the discrete levels. The experimental data seemed to confirm this model [7] based on the quantized one-dimesional Josephson washboard potential. This quantum picture was embraced by the community of researchers interested in the possibility of using Josephson based circuits as superconducting qubits.

However, no classical considerations were made in [5], so the argument as to an applicable model was in truth incomplete. In fact, as we show here, the classical RCSJ model, which is the foundation for the above-mentioned quantum phenomenology, with dc bias and applied microwaves predicts the experimental observations to high accuracy.

The phase dynamics of a Josephson junction which is biased with both dc and ac currents are governed by the simple classical expression [1]

$$
\ddot{\varphi}+\alpha \dot{\varphi}+\sin \varphi=\eta+\eta_{d} \sin \left(\Omega_{d} \tau\right),
$$

where the normalized dissipation is $\alpha=\hbar \omega_{0} / 2 e R I_{c}$, with the Josephson zero-bias plasma frequency $\omega_{0}=$ $\sqrt{2 e I_{c} / \hbar C}$. The junction RCSJ parameters are: critical current $I_{c}$, capacitance $C$, resistance $R$. In this equation, the fixed and ac bias currents $\left(\eta\right.$ and $\eta_{d}$ ) have been normalized to $I_{c}$, the ac frequency has been normalized to $\omega_{0}$, and time is in units of $\omega_{0}^{-1}$. Equation (11) can be viewed as describing the classical motion of a fictitious particle on a one dimensional surface

$$
U(\varphi)=-\eta \varphi-\cos \varphi,
$$

with the potential expressed in units of the Josephson coupling energy $E_{J}=\hbar I_{c} / 2 e$. This washboard potential has a repeated series of wells which become shallower as the bias $\eta$ is increased. The natural frequency of small oscillations at the bottom of each well changes with bias according to $\omega_{p}=\omega_{0}\left(1-\eta^{2}\right)^{\frac{1}{4}}$. The position of the well minimum is $\varphi_{\min }=\sin ^{-1} \eta$ and the rim is located at $\varphi_{\text {crit }}=\pi-\varphi_{\min }$.

In the absence of ac excitation $\left(\eta_{d}=0\right.$ in Eq.(10), the particle would sit at the bottom of the well. Sufficient additional thermal noise can activate escape over 

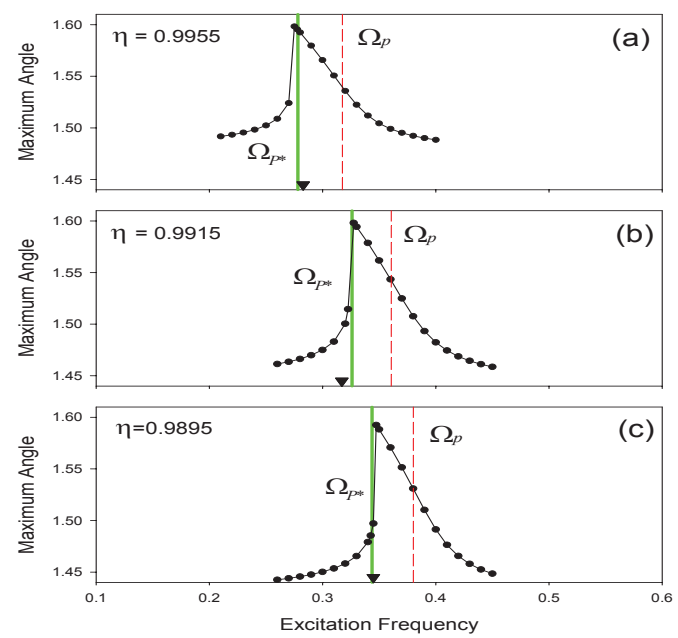

FIG. 1: Plots of maximum amplitude of the junction phase oscillation on the barrier side of the potential well as a function of the normalized frequency of the applied microwave excitation for three different normalized bias currents $\eta$ (numerical simulation). In each case, the bias dependent normalized plasma frequency, determined from the expression $\Omega_{P}=\left(1-\eta^{2}\right)^{\frac{1}{4}}$, is shown by a dashed vertical line. The anharmonic result $\Omega_{P *}$ from Eq.(3) is shown by a solid vertical line. The normalized microwave frequencies from the experiments described in [5] are marked by inverted black triangles.

the barrier $\Delta U=2\left[\sqrt{1-\eta^{2}}-\eta \cos ^{-1} \eta\right]$. Resonant activation $\left(\eta_{d}>0\right)$ provides an alternate mechanism for escape, but the anharmonic shape of the well means that the ideal condition is not simply to set the normalized forcing frequency $\Omega_{d}$ to $\left(1-\eta^{2}\right)^{\frac{1}{4}}$; generally speaking a slightly lower value is optimum. It is also true that when the forcing frequency is properly tuned to a specific well, there will be some forcing amplitude above which escape will always occur. This means that just below such a drive threshold and when combined with even very small thermal noise, stochastic activation will occur, as is seen in all the experiments.

A simple anharmonic analysis of the classical model can produce explicit results that can be compared directly with experiments. This was first done in Refs. 8 , 9], where direct comparisons between classical theory and experiments showed that microwave-induced resonant switching can be understood classically. The key results of the analysis come from introducing a monochromatic ansatz $\varphi=\varphi_{0}+a \sin \Omega_{p *} \tau$ ( $\varphi_{0}$ is a constant and $a$ is the oscillation amplitude) into Eq.(1) for $\alpha=\eta_{d}=0$. This yields the effective relationships $J_{0}(a) \sin \varphi_{0}=\eta$ and

$$
\Omega_{p *}^{2}=\frac{2 J_{1}(a)}{a} \sqrt{1-\left(\frac{\eta}{J_{0}(a)}\right)^{2}},
$$

where $J_{n}$ is the Bessel function of $n$th order and first kind. Since the experiments are conducted such that high escape probability exists near the sought-after resonance while vanishing escape probability should exist when the system is off-resonance, the strength of the microwave field should be such that the resulting oscillation amplitude reaches the inflection point of the effective potential; i.e.,

$$
a=\frac{\pi}{2}-\sin ^{-1} \frac{\eta}{J_{0}(a)} .
$$

Oscillating with this amplitude will result in a good statistical probability that the junction switches to the nonzero voltage state within some allowed time. A useful explicit second order approximation in $a$ for $\eta \rightarrow 1$ to the desired amplitude is given by $a^{2}=\frac{4}{3}(1-\eta)$. Inserting this result into Eq.(3) provides an explicit relationship between the anharmonic classical resonance and the bias current for direct comparisons with reported experiments in the limit of validity for Eq.(44). The pro-

\begin{tabular}{|l|l|l|l|l|}
\hline Figure & $\begin{array}{l}\text { Frequency } \\
(\mathrm{GHz})\end{array}$ & $\begin{array}{l}\text { Bias } \\
(\mu \mathrm{A})\end{array}$ & $\Omega_{d}$ & $\eta$ \\
\hline 2(a) & 2.0 & 30.435 & 0.283 & 0.9955 \\
\hline $3(\mathrm{a})$ & 3.4 & 9.408 & 0.317 & 0.9915 \\
\hline $3(\mathrm{a})$ & 3.5 & 9.402 & 0.326 & 0.9908 \\
\hline $3(\mathrm{a})$ & 3.6 & 9.397 & 0.336 & 0.9902 \\
\hline $3(\mathrm{a})$ & 3.7 & 9.388 & 0.345 & 0.9895 \\
\hline
\end{tabular}

TABLE I: Summary of experimental data for the peaks in the escape rates as reported in Martinis et al. [5].

tocol adopted in the experiments [5, 8] was to fix the microwave frequency and then observe the escape rate while scanning the bias current. Our attention here is on the results originally presented in [5], most particularly the data in their Figs. 2 and 3. Two different physical samples were used - the parameters for Fig.2 were given as $I_{c}=30.572 \mu \mathrm{A}, C=47.0 \mathrm{pF}$, yielding a plasma frequency $\omega_{0}=4.443 \times 10^{10} \mathrm{~s}^{-1}$, whereas for Fig. 3 they were $I_{c}=9.489 \mu \mathrm{A}, C=6.35 \mathrm{pF}$, giving a plasma frequency $\omega_{0}=6.734 \times 10^{10} s^{-1}$. From blowups of these figures, it was possible to identify the peak positions, that is the bias current at maximum escape rate for each stated microwave frequency - these are given in the second and third columns of Table I. Normalized values $\Omega_{d}$ and $\eta$ are listed in the fourth and fifth columns. The most prominent peak in Fig. 2 and all four peaks in Fig. 3 are included. It should be noted that because the precise shapes of the potential wells are completely determined by the bias current $\eta$, it is somewhat more physically meaningful, in contrast to the experimental protocol mentioned above, to fix the bias current and vary the excitation frequency. This approach reveals the optimum excitation frequency for any given well (bias). Such a strategy was followed in carrying out numerical solutions of Eq.(11). In these simulations, the value of the dissipation constant $\alpha$ was chosen to be 0.00845 , otherwise there were no adjustable parameters. For any selected bias $\eta$ matching one of the experimental values, a series of runs was carried out for specific frequencies $\Omega_{d}$. The excitation amplitude $\eta_{d}$ was ramped up from zero to its final value (typically in the range 0.0007 to 0.001 ) over 


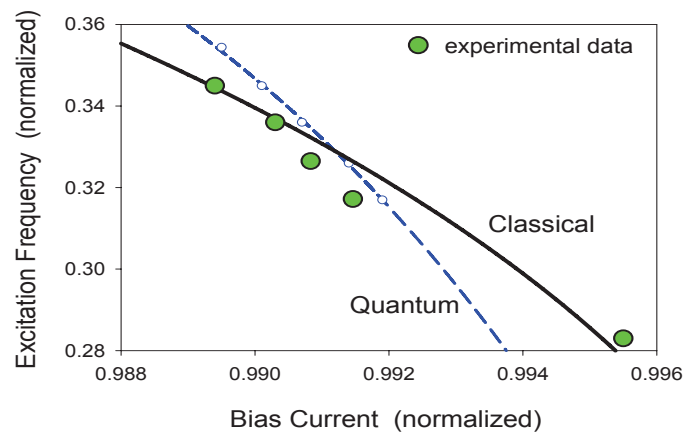

FIG. 2: A comparison of the classical and quantum models for the escape to a finite voltage state of a low temperature Josephson junction biased very close to its critical current and with applied microwaves. The 5 experimental data points are from Martinis et al. 5], as is their predicted quantum result (dashed line). The anharmonic analysis of the classical model yields the solid line.

an interval of 600 plasma periods; this was done to avoid spurious start up effects. An additional 400 plasma periods were then recorded. In each run the resulting data set from the numerical solution $\varphi(\tau)$ was examined for the largest repetitive maximum angle reached. These maximum angles are plotted in the three panels of Fig.1. They correspond to the first, second and fifth rows of Table I. Computations corresponding to the other bias values yield the same general behavior. The obvious asymmetries of the responses to ac excitation are a result of the anharmonicity of the wells. The inverted filled triangles mark the frequencies of the experimentally determined peaks in the escape rates, as reported in [5]. These frequencies coincide almost exactly with the location of the sudden onset of large induced phase oscillations in the numerical solutions of Eq.(1). These plots also reveal that good agreement should not be expected with the normalized linear plasma frequency $\Omega_{p}$ because it is always an over-estimate of the true optimum excitation for which a better expression is given by Eqs. (3) and (4).

Figure 2 presents a direct comparison of the classical and quantum models with respect to the experimental data reported in [5]. The left-most four points come from the four peaks presented in Fig. 3(a) of [5]; the fifth data point, on the far right, comes from the position of the right-most peak in Fig. 2(a) of [5]. The dashed line labeled "Quantum" is obtained from the predicted energy level spacing $E_{0 \rightarrow 1}$ shown as a solid curve in Fig. 3(b) of [5]; the solid curve labeled "Classical" is generated from Eqs. (3) and (4).

As has been demonstrated, in the absence of noise, optimum ac excitation will induce a maximum excursion in the phase of the Josephson junction. These largest excursions take the system closest to the critical value $\varphi_{\text {crit }}$, at which escape would occur. Our basic hypothesis is simple: for a slightly subcritical excitation, additional noise will stochastically induce escapes, as was

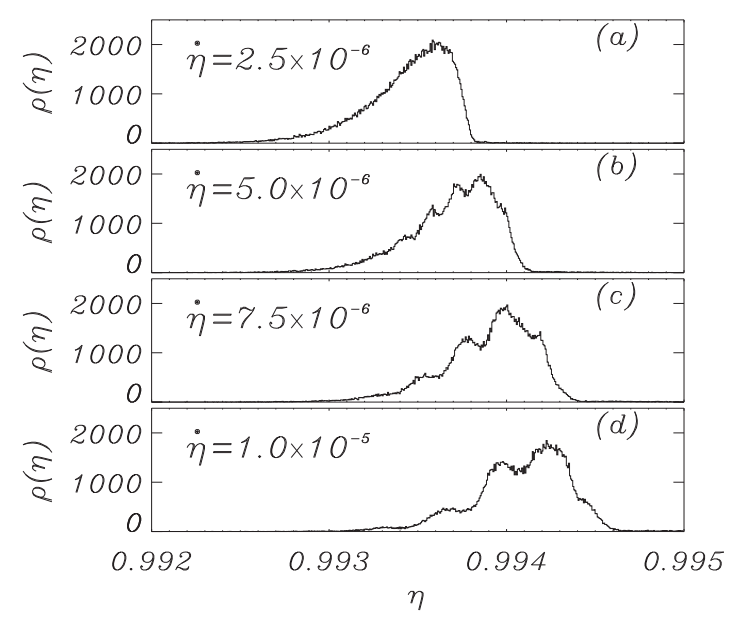

FIG. 3: Switching distributions for swept bias $\eta$. For these simulations, we chose $k_{B} T / E_{J}=3.8418 \times 10^{-5}$ and $\Omega_{d}=$ 0.282809 to match the $2 \mathrm{GHz}$ frequency in Fig. 2 of [5]. Each distribution is generated from 100,000 switching events, and each simulation is initiated at $\eta=0$. The applied sweep rate, microwave amplitude and damping were not reported in [5]; we have chosen $\alpha=10^{-4}$ and $\eta_{d}=0.0019$. The applied sweep rate $\dot{\eta}$ is reported on each panel.

demonstrated in [9]. Therefore the experimental observations of peaks in the escape rates at finite temperature are fully consistent with the classical model embodied in Eq. (11). Agreement between predictions of the classical model with experimental values for the dc bias $(\eta)$ and microwave frequency $\left(\Omega_{d}\right)$ at these peaks is very accurate. Moreover, the classical model maintains its accuracy over two distinct experimental samples having quite different critical currents and capacitances. One curious observation made in [5] is the appearance of multiple resonant escape peaks seen in their Figure 2. These were interpreted as evidence for the higher quantum transitions $1 \rightarrow 2,2 \rightarrow 3$, and so on. However, no signatures of these were seen in the comparable escape probability plots shown in their Figure 3. We further notice that such a family of peaks was also not observed in [10]. Our simulations of the classical model show that closely spaced multiple resonant escape peaks can be observed under certain conditions due to the nonlinearity that provides the possibility for phase-locking of an oscillator at many harmonics (sub, super, and fractional). We mention in passing that this has also been shown [8] to explain the so-called multi-photon absorption peaks discussed in [10]. One could imagine that the multiple resonances seen in Figure 2 of [5] could be due to some fractional resonance, but we see no evidence for this in the classical model for the given parameters. Instead, we have noticed very similar families of resonant peaks appearing when the bias current sweep rate is elevated. This important parameter is not given in [5], but typical values would correspond to a normalized sweep rate in the interval between $\sim 10^{-8}$ 
and $\sim 10^{-5}$. It is in the upper end of this range (faster sweep rates) that we encounter the appearance of the multiple peaks similar to those observed in [5]. Figure 3 shows the appearance of such a switching peak family as a function of the bias sweep rate $\dot{\eta}$ for a fixed microwave amplitude . All other parameters are chosen according to the experiments leading to figure 2 in [5]. Thus we included in the right hand side of Eq.(11) a stochastic current noise term [8, 9] corresponding to the thermodynamic temperature of $28 \mathrm{mK}$; each distribution in Fig. 3 is the result of 100,000 switching events after initiating the system at $\eta=0$.

We clearly observe in Fig. 3 the emergence of multiple peaks, and we further observe that the spacing between the peaks increases with the sweep rate. We have investigated the details of the resonance near the bias values of the multiple peaks, and we have found only a single resonance, which is well described by Eqs. (3) and (4), and this is consistent with the fact that only a single resonant peak is found for small sweep rates. Thus, the multiplicity of apparent resonant peaks must be related to an interference between the microwave oscillation and the non-negligible variation in $\eta$ for high sweep rates. In this context it is worthwhile recalling that a fast bias current sweep rate has previously been shown to produce multi-peak switching distributions in simulations of long Josephson junctions [11], and it has been shown experimentally that fast sweep rates can produce multiple peaks in the conditional switching distribution [12] even without the application of microwaves.

To conclude, we have established that the postulated quantum ingredients of discrete levels and macroscopic quantum tunneling are superfluous in explaining the overall results of experiments on resonant escape in current biased Josephson junctions at low temperature and with applied microwaves; the well-known classical model has been shown to yield excellent agreement with the reported experimental data. This agreement is consistent with the ones previously reported in Refs. [8, 9], and is quite similar to our recent examination of claims of quantum entanglement in coupled Josephson qubits [13], and to our earlier work [14] showing that the classical model accounts for reports of, e.g., Rabi oscillations in large Josephson junction qubits [15].

The often repeated claim [16] that the phase variable of Josephson junctions in these superconducting systems at these temperatures has been "unequivocally" demonstrated to be exclusively a quantum variable is not supported by the present evidence. What is lacking are experimental observations of phenomena that cannot be encompassed within a classical picture and thus require an exclusively quantum model. The classical RCSJ model seems adequate in seeking an overall understanding of these types of experiment where microwaves are applied to Josephson systems.

\section{Acknowledgments}

This work was supported in part (JAB) by a grant from the Natural Sciences and Engineering Research Council of Canada.
[1] T. Van Duzer and C.W. Turner, Principles of Superconductive Devices and Circuits,(second ed.) Prentice Hall PTR Upper Saddle River, N.J. USA (1998), Chapter 5

[2] R.F. Voss and R.A. Webb, Phys. Rev. Lett. 47, 265 (1981).

[3] I. Affleck, Phys. Rev. Lett. 46, 388 (1981)

[4] M.H. Devoret, J.M. Martinis, and J. Clarke, Phys. Rev. Lett. 55, 1908 (1985)

[5] J.M. Martinis, M.H. Devoret, and J. Clarke, Phys. Rev. Lett. 55, 1543 (1985)

[6] J.M. Martinis, M.H. Devoret, and J. Clarke, Phys. Rev. B 35, 4682 (1987)

[7] Of course, if the temperature is not exceptionally low, then one is in a classical regime; it should be noted that similar experiments carried out above $1 K$ have always been treated as examples of resonant activation. See: M.H. Devoret, J.M. Martinis, D. Esteve, and J. Clarke Phys. Rev. Lett. 53, 1260 (1984) and M.H. Devoret, D. Esteve, J.M. Martinis, A. Cleland, and J.Clarke, Phys. Rev. B 36, 58 (1987).

[8] N. Grønbech-Jensen, M.G. Castellano, F. Chiarello, M. Cirillo, C. Cosmelli, I.V. Filippenko, R. Russo, and G. Torrioli, Phys. Rev. Lett. 93, 107002 (2004)

[9] "Anomalous thermal escape in Josephson systems per- turbed by microwaves", N. Grønbech-Jensen, M.G. Castellano, F. Chiarello, M. Cirillo, C. Cosmelli, V. Merlo, R. Russo, and G. Torrioli, in Quantum Computing: Solid State Systems, pp. 111-119 (2006). Eds. B. Ruggeiro, P. Delsing, C. Granata, Y. Paskin, and P. Silvestrini, Kluwer Academic/Plenum Publishers (Springer, N.Y. 2006). ISBN-10: 0-387-26332-2; ISBN-13: 9780387-26332-8; cond-mat/0412692

[10] A. Wallraff, T. Duty, A. Lukashenko, and A.V. Ustinov, Phys. Rev. Lett. 90, 037003 (2003)

[11] N. Grønbech-Jensen and M. Cirillo, Phys. Rev. B 70, 214507 (2004)

[12] P. Silvestrini, V.G. Palmieri, B. Ruggiero, and M. Russo, Phys. Rev. Lett. 79, 3046 (1997)

[13] J.A. Blackburn, J.E. Marchese, M. Cirillo, and N. Grønbech-Jensen, Phys. Rev. B 79, 054516 (2009)

[14] N. Grønbech-Jensen and M. Cirillo, Phys. Rev. Lett. 95, 067001 (2005); J.E. Marchese, M. Cirillo, and N. Grønbech-Jensen, Phys. Rev. B 73, 174507 (2006)

[15] J.M. Martinis, S. Nam, J. Aumentado, and C. Urbina, Phys. Rev. Lett. 89, 117901 (2002)

[16] J. Clarke and F.K. Wilhelm, Nature 453, 1031 (2008) 